\title{
Common drugs, vitamins, nutritional supplements and COVID-19 mortality
}

\author{
STEVEN LEHRER ${ }^{1}$ and PETER H. RHEINSTEIN ${ }^{2}$ \\ ${ }^{1}$ Department of Radiation Oncology, Icahn School of Medicine at Mount Sinai, New York, NY 10029; \\ ${ }^{2}$ Severn Health Solutions, Severna Park, MD 21146, USA
}

Received January 19, 2021; Accepted February 25, 2021

DOI: $10.3892 /$ ijfn.2021.14

\begin{abstract}
The FDA has approved only one drug, remdesivir, for the treatment of COVID-19. The FDA has granted an emergency use authorization for the rheumatoid arthritis treatment drug, baricitinib (Olumiant), for the treatment of COVID-19 in some cases. For this reason, investigators have paid considerable attention to the association between commonly used drugs and the outcome of patients with COVID-19. Aspirin and ibuprofen have been reported to reduce the mortality rate. Omeprazole can increase mortality. In addition, some studies have demonstrated that famotidine diminishes mortality, while others have indicated that famotidine leads to a poorer prognosis. The present study used UK Biobank (UKB) data to assess the association of commonly used drugs with COVID-19 mortality. Data processing was performed on Minerva, a Linux mainframe with Centos 7.6. The UK Biobank Data Parser (ukbb_parser) was used, a python-based package that allows easy interfacing with the large UK Biobank dataset. The results revealed that aspirin and omeprazole were associated with an elevated mortality rate. Ibuprofen-related mortality was lower than laxative-related mortality. Aspirin users were also significantly older than other subjects. The association with mortality of cholesterol-lowering medications, blood pressure-lowering medications, hormone replacement and oral contraceptives in 134 female subjects revealed insignificant variability. The association of nutritional supplements in 238 subjects with mortality indicated that variability was insignificant. The lower mortality linked to the supplementation of vitamin D and vitamin B, presumably B complex, has been previously observed. On the whole, the present study demonstrates that although some of the associations described among drugs and COVID-19 are not novel, the utility of a new source, UKB, may prove to be useful in further examining these associations.
\end{abstract}

Correspondence to: Dr Steven Lehrer, Department of Radiation Oncology, Icahn School of Medicine at Mount Sinai, 1 Gustave L. Levy Place, New York, NY 10029, USA

E-mail: steven.lehrer@mssm.edu

Key words: drugs, COVID-19, mortality

\section{Introduction}

Therapy for COVID-19, particularly during the early stages of infection, is urgently required. Although COVID-19 is mostly self-limited, up to $20 \%$ of symptomatic cases will develop severe or critical disease with clinical manifestations, including pneumonia, acute respiratory distress syndrome, multiorgan system dysfunction, hypercoagulation and hyperinflammatory manifestations. Over 47 million cases of COVID-19 globally have resulted in $>1.2$ million deaths (1).

The FDA has approved only one drug, remdesivir, for the treatment of COVID-19. In addition, the FDA has granted an emergency use authorization for the use of baricitinib (Olumiant; used for the treatment of rheumatoid arthritis) for the treatment of COVID-19 in some cases. For this reason, investigators have paid considerable attention to the association between the administration of commonly used drugs with the outcome of patients with COVID-19. Aspirin (2-5) has been reported to reduce mortality. However, as regards other drugs, some studies have recommended that ibuprofen should be avoided (6-8). In addition, the use of omeprazole has been shown to increase mortality (9). At least one report has indicted that famotidine decreases mortality (10), while another indicates that the use of famotidine leads to a poorer prognosis (11).

\section{Data and methods}

UK Biobank (UKB). UKB data were used to assess the association of common drugs with COVID-19 mortality. Electronic linkage between UKB records and National Health Service COVID-19 laboratory test results in the UK were available from March 16, to April 26, 2020, including the peak of daily COVID-19 laboratory-confirmed cases. During this time period, the testing of older age groups was largely restricted to hospital inpatients exhibiting clinical signs of infection. Thus, test positivity is considered a good marker of severe COVID-19 (12). COVID-19 mortality in the UK was recorded as 900 deaths per day at this time.

For the data used in the present study, the inclusion criteria were a positive test for COVID-19, and the exclusion criteria were negative COVID-19 test or no test results.

Data processing and statistical analysis. Data processing was performed on Minerva, a Linux mainframe with Centos 7.6, 
at the Icahn School of Medicine at Mount Sinai. The UKB Data Parser (ukbb_parser) was used, which is a python-based package that allows easy interfacing with the large UK Biobank dataset (13). Data were analyzed using one-way analysis of variance (ANOVA), Tukey's post hoc test, logistic regression, and Fisher's exact test. Statistical analysis was performed using SPSS version 22.

\section{Results}

The present study evaluated data from 376 subjects, $49 \%$ female, $51 \%$ male. The age of the subjects was $55.7 \pm 9.1$ (mean \pm SD) years. The COVID-19 mortality rate was $20.5 \%$.

The association between the use of aspirin, ibuprofen, paracetamol (acetaminophen), ranitidine, omeprazole and laxatives with mortality in 376 subjects, 517 responses is shown in Table I. The variability was significant $(\mathrm{P}=0.011$, two-tailed Fisher's exact test).

As regards the use of Fisher's exact test, it should be noted that this is a statistical test used to determine whether there are non-random associations between categorical variables. Only in Table I does such an association exist, suggesting that the variation in mortality rates of aspirin, ibuprofen, paracetamol (acetaminophen), ranitidine, omeprazole and laxatives is meaningful. In the other tables, variability was not significant, suggesting that the differing mortalities may not be meaningful. Given the large number of agents examined, the present study performed as few comparison tests as possible. When performing hypothesis tests with multiple comparisons, eventually a result could occur that appears to demonstrate statistical significance in the dependent variable, even when there is none.

Aspirin and omeprazole were associated with an increased mortality. Ibuprofen-related mortality was lower than laxative-related mortality. Aspirin users were significantly older $(\mathrm{P}<0.001$, one-way ANOVA; Fig. 1).

The association with mortality of cholesterol-lowering medications, blood pressure-lowering medications, insulin, hormone replacement and oral contraceptives in 134 female subjects and 173 responses is shown in Table II. The variability was insignificant $(\mathrm{P}=0.077$, two-tailed Fisher's exact test). The age (mean $\pm \mathrm{SD}$ ) of the female subjects by medication are shown in Fig. 2. Oral contraceptive users were significantly younger than hormone replacement users $(\mathrm{P}<0.05$, Tukey's range test). The hormone replacement users were significantly younger than the users of cholesterol-lowering medications and blood pressure-lowering medications $(\mathrm{P}<0.05$, Tukey's range test). The effect of age on survival was significant; however, the effect of hormones on survival was not (Table III).

The association of nutritional supplements with mortality is shown in Table IV. The lower mortality linked to vitamin D and vitamin B supplementation, presumably B complex, has been previously observed $(14,15)$. There was no significant difference in the mean age $(\mathrm{P}=0.071$, one-way ANOVA; data not shown).

The association of mineral and other dietary supplements with mortality, in 294 subjects, 456 responses is shown in Table V. The variability was insignificant $(\mathrm{P}=0.983$, two-tailed Fisher's exact test).
Table I. Association of aspirin, ibuprofen, paracetamol (acetaminophen), ranitidine, omeprazole and laxatives with mortality.

\begin{tabular}{llccc}
\hline Drug & Count and \% & Alive & Deceased & Total \\
\hline Aspirin & Count & 84 & 35 & 119 \\
& $\%$ With drug use & $70.60 \%$ & $29.40 \%$ & \\
Ibuprofen & Count & 106 & 15 & 121 \\
& $\%$ With drug use & $87.60 \%$ & $12.40 \%$ & \\
Paracetamol & Count & 150 & 34 & 184 \\
& $\%$ With drug use & $81.50 \%$ & $18.50 \%$ & \\
Ranitidine & Count & 11 & 4 & 15 \\
& $\%$ With drug use & $73.30 \%$ & $26.70 \%$ & \\
Omeprazole & Count & 38 & 14 & 52 \\
& $\%$ With drug use & $73.10 \%$ & $26.90 \%$ & \\
Laxatives & Count & 18 & 8 & 26 \\
& $\%$ With drug use & $69.20 \%$ & $30.80 \%$ & \\
No. of subjects & Count & 299 & 77 & 376 \\
\hline
\end{tabular}

There were 517 responses, 376 subjects. The variability is significant ( $\mathrm{P}=0.011$, two-tailed Fisher exact test). Aspirin and omeprazole have elevated mortality. Ibuprofen mortality $(12.4 \%)$ is lower than laxative mortality $(30.8 \%)$.

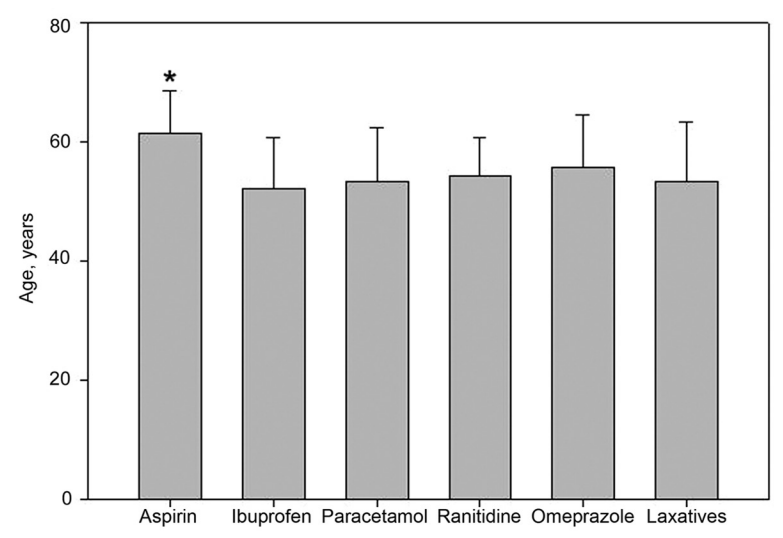

Figure 1. Age (mean \pm SD) of subjects, both sexes, by medication. Aspirin users were significantly older $\left({ }^{*} \mathrm{P}<0.001\right.$, one-way analysis of variance).

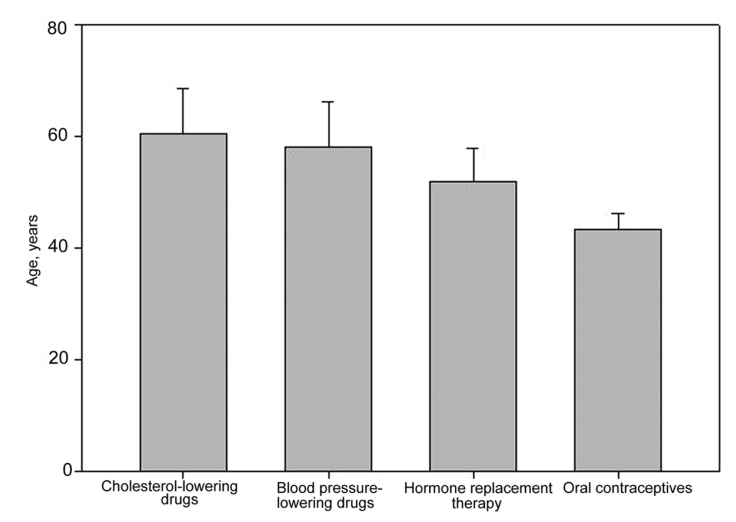

Figure 2. Age (mean $\pm \mathrm{SD}$ ) of female subjects by medication. Oral contraceptive users were significantly younger than hormone replacement users $(\mathrm{P}<0.05$, Tukey's range test). The hormone replacement users were significantly younger than users of cholesterol lowering medications and blood pressure lowering medications $(\mathrm{P}<0.05$, Tukey's range test). There were no age-related data available for insulin users. 
Table II. Association with mortality of cholesterol lowering medications, blood pressure lowering medications, insulin, hormone replacement and oral contraceptives.

\begin{tabular}{|c|c|c|c|c|c|}
\hline Sex & Type of drug & Count and \% & Alive & Deceased & Total \\
\hline \multirow[t]{10}{*}{ Female } & Cholesterol-lowering & Count & 35 & 12 & 47 \\
\hline & & $\%$ With drug use & $74.50 \%$ & $25.50 \%$ & \\
\hline & Blood pressure-lowering & Count & 53 & 16 & 69 \\
\hline & & $\%$ With drug use & $76.80 \%$ & $23.20 \%$ & \\
\hline & Insulin therapy & Count & 5 & 2 & 7 \\
\hline & & $\%$ With drug use & $71.40 \%$ & $28.60 \%$ & \\
\hline & Hormone replacement therapy & Count & 29 & 2 & 31 \\
\hline & & $\%$ With drug use & $93.50 \%$ & $6.50 \%$ & \\
\hline & Oral contraceptives & Count & 17 & 1 & 19 \\
\hline & & $\%$ With drug use & $94.40 \%$ & $5.60 \%$ & \\
\hline No. of females & & Count & 103 & 21 & 134 \\
\hline
\end{tabular}

The association was examined in 134 female subjects, 173 responses. The variability was insignificant $(\mathrm{P}=0.077$, two-tailed Fisher's exact test).

Table III. Effect age and hormones on survival.

\begin{tabular}{lcccr}
\hline & $95 \% \mathrm{LB}$ & OR & $95 \% \mathrm{UB}$ & P-value \\
\hline Age & 1.149 & 1.188 & 1.228 & $<0.001$ \\
Hormones & 0.102 & 0.477 & 2.240 & 0.348 \\
\hline
\end{tabular}

Logistic regression was performed for hormone use (yes or no) and age, independent variables, and alive or deceased dependent variable. ORs, $95 \%$ confidence intervals (LB and UB) are shown. The effect of age on survival was significant $(\mathrm{P}<0.001)$ with survival decreased by a factor of 1.88 with each year of age. The effect of hormone use was not significant $(\mathrm{P}=0.348)$. OR, odds ratio; LB, lower bound; $\mathrm{UB}$, upper bound.

Table IV. Association of nutritional supplements with mortality.

\begin{tabular}{llccc}
\hline Supplement & Count and \% & Alive & Deceased & Total \\
\hline Vitamin A & Count & 14 & 3 & 17 \\
& $\%$ With drug use & $82.40 \%$ & $17.60 \%$ & \\
Vitamin B & Count & 31 & 5 & 36 \\
& $\%$ With drug use & $86.10 \%$ & $13.90 \%$ & \\
Vitamin C & Count & 50 & 15 & 65 \\
& $\%$ With drug use & $76.90 \%$ & $23.10 \%$ & \\
Vitamin D & Count & 26 & 3 & 29 \\
& $\%$ With drug use & $89.70 \%$ & $10.30 \%$ & \\
Vitamin E & Count & 21 & 3 & 24 \\
\multirow{2}{*}{ Folate } & $\%$ With drug use & $87.50 \%$ & $12.50 \%$ & \\
& Count & 17 & 5 & 22 \\
Multivitamins & Count & $77.30 \%$ & $22.70 \%$ & \\
& $\%$ With drug use & $78.50 \%$ & $21.50 \%$ & \\
Total no. of & Count & 189 & 49 & 238 \\
subjects & & & & \\
\hline
\end{tabular}

Data are shown for 238 subjects, 356 responses. Variability was insignificant. The lower mortality associated with vitamin $\mathrm{D}$ and vitamin $\mathrm{B}$ (presumably B complex) has been previously observed $(13,14)$. The variability was insignificant $(\mathrm{P}=0.696$, two-tailed Fisher's exact test).
Table V. Association of mineral and other dietary supplements with mortality.

\begin{tabular}{llccc}
\hline Supplement & Count and \% & Alive & Deceased & Total \\
\hline $\begin{array}{l}\text { Fish oil } \\
\text { (including }\end{array}$ & Count & 166 & 40 & 206 \\
cod liver oil) & & & & \\
Glucosamine & Count & 97 & 27 & 124 \\
& $\%$ With drug use & $78.20 \%$ & $21.80 \%$ & \\
Calcium & Count & 39 & 11 & 50 \\
& $\%$ With drug use & $78.00 \%$ & $22.00 \%$ & \\
Zinc & Count & 28 & 6 & 34 \\
& $\%$ With drug use & $82.40 \%$ & $17.60 \%$ & \\
Iron & Count & 20 & 6 & 26 \\
& $\%$ With drug use & $76.90 \%$ & $23.10 \%$ & \\
Selenium & Count & 13 & 3 & 16 \\
& $\%$ With drug use & $81.30 \%$ & $18.80 \%$ & \\
Total no. of & Count & 238 & 56 & 294 \\
subjects & & & & \\
\hline
\end{tabular}

Data are shown for 294 subjects, 456 responses. The variability was insignificant ( $\mathrm{P}=0.983$, two-tailed Fisher's exact test).

\section{Discussion}

In hospitalized UKB COVID-19 patients, common comorbidities are hypertension, coronary heart disease, atrial fibrillation, type 2 diabetes and dementia. All these conditions are associated with an increased mortality (12).

Coagulopathy and blood clots are a significant adverse event in patients with COVID-19. Aspirin inhibits the coagulation due to its effect on platelets and has been reported to improve patient prognosis (3-5). The present study was not able to confirm this observation as the patients on aspirin in the current sample were significantly older and therefore less 
likely to survive. Many patients were no doubt using aspirin for cardioprotection and/or arthritis. Clinical trials, as for example, the FREEDOM-COVID study (ClinicalTrials.gov identifier: NCT04512079), are attempting to determine the best anticoagulant strategy for patients admitted to general medical wards. The ranitidine bismuth citrate combination has been shown to suppress SARS-CoV-2 replication and to relieve virus-associated pneumonia in Syrian hamsters (16); however, this effect may not occur in patients with COVID-19 taking ranitidine. In the present study, ibuprofen-related mortality (12.4\%) was lower than laxative-related mortality (30.8\%), suggesting that ibuprofen does not worsen the clinical outcome $(7,8)$.

Cholesterol-lowering medications, blood-pressure lowering medications, hormone replacement therapy and oral contraceptives seem to have minimal effects on patient outcome. The younger age of subjects on hormone replacement therapy and oral contraceptive users most probably explains their reduced mortality.

Nutritional supplements may increase survival. Mortality in an Italian nursing home during the COVID-19 pandemic was shown to be reduced with vitamin D supplementation (14). A vitamin D/magnesium/vitamin B12 combination in older-aged COVID-19 patients was associated with a significant reduction in the proportion of patients with clinical deterioration requiring oxygen support, intensive care support, or both (15). Vitamin D may be a critical host factor to prevent COVID-19 (17), although this hypothesis remains controversial $(18,19)$. To prevent the cytokine storm, the use of $\mathrm{N}$-acetylcysteine in both the prevention and adjuvant therapy of COVID-19 has been suggested; however, the UKB did not have information on this substance (20).

In conclusion, although some of the associations described herein among drugs and COVID-19 are not novel, the present study demonstrates the utility of a new source, the UKB, in further examining these associations. The association of lifestyle, comorbidities, medication and phenotypic information with patient outcomes will become an invaluable source as more UKB data become available on subjects tested for COVID-19 (21).

\section{Acknowledgements}

This work was supported in part through the computational resources and staff expertise provided by Scientific Computing at the Icahn School of Medicine at Mount Sinai.

\section{Funding}

The research reported in the present study was supported by the Office of Research Infrastructure of the National Institutes of Health under award nos. S10OD018522 and S10OD026880. The content is solely the responsibility of the authors and does not necessarily represent the official views of the National Institutes of Health.

\section{Availability of data and materials}

All data generated or analyzed during this study are included in this published article or are held by UK Biobank.

\section{Authors' contributions}

SL and PHR contributed equally to the conception, writing and data analysis of the present study.

\section{Ethics approval and consent to participate}

All patient data were from the UK Biobank. The UK Biobank application for the present study was approved as UKB project 57245, SL and PHR.

\section{Patient consent for publication}

Not applicable.

\section{Competing interests}

The authors declare that they have no competing interests.

\section{References}

1. Kim PS, Read SW and Fauci AS: Therapy for early COVID-19: A critical need. JAMA 324: 2149-2150, 2020.

2. Chow JH, Khanna AK, Kethireddy S, Yamane D, Levine A, Jackson AM, McCurdy MT, Tabatabai A, Kumar G, Park P, et al: Aspirin use is associated with decreased mechanical ventilation, ICU admission, and in-hospital mortality in hospitalized patients with COVID-19. Anesth Analg: Oct 21, 2020 (Epub ahead of print). doi: 10.1213/ANE.0000000000005292.

3. Haque S, Jawed A, Akhter N, Dar SA, Khan F, Mandal RK, Areeshi MY, Lohani M and Wahid M: Acetylsalicylic acid (Aspirin): A potent medicine for preventing COVID-19 deaths caused by thrombosis and pulmonary embolism. Eur Rev Med Pharmacol Sci 24: 9244-9245, 2020.

4. Mohamed-Hussein AA, Aly KM and Ibrahim MA: Should aspirin be used for prophylaxis of COVID-19-induced coagulopathy? Med Hypotheses 144: 109975, 2020.

5. Pavoni V, Gianesello L, Pazzi M, Stera C, Meconi T and Frigieri FC: Venous thromboembolism and bleeding in critically ill COVID-19 patients treated with higher than standard low molecular weight heparin doses and aspirin: A call to action. Thromb Res 196: 313-317, 2020.

6. Kelleni MT: Early use of non-steroidal anti-inflammatory drugs in COVID-19 might reverse pathogenesis, prevent complications and improve clinical outcomes. Biomed Pharmacother 133: $110982,2021$.

7. Kutti Sridharan G, Kotagiri R, Chandiramani VH, Mohan BP, Vegunta R, Vegunta R and Rokkam VR: COVID-19 and avoiding Ibuprofen. How good is the evidence? Am J Ther 27: e400-e402, 2020.

8. Rinott E, Kozer E, Shapira Y, Bar-Haim A and Youngster I: Ibuprofen use and clinical outcomes in COVID-19 patients. Clin Microbiol Infect 26: 1259.e5-1259.e7, 2020.

9. Lee SW, Ha EK, Yeniova AÖ, Moon SY, Kim SY, Koh HY, Yang JM, Jeong SJ, Moon SJ, Cho JY, et al: Severe clinical outcomes of COVID-19 associated with proton pump inhibitors: A nationwide cohort study with propensity score matching. Gut 70: 76-84, 2021.

10. Mather JF, Seip RL and McKay RG: Impact of famotidine use on clinical outcomes of hospitalized patients with COVID-19. Am J Gastroenterol 115: 1617-1623, 2020.

11. Zhou J, Wang X, Lee S, Wu WK, Cheung BM, Zhang Q and Tse G: Proton pump inhibitor or famotidine use and severe COVID-19 disease: A propensity score-matched territory-wide study. Gut: 2020, 323668, 2020.

12. Atkins JL, Masoli JA, Delgado J, Pilling LC, Kuo CL, Kuchel GA and Melzer D: Preexisting comorbidities predicting COVID-19 and mortality in the UK Biobank community cohort. J Gerontol A Biol Sci Med Sci 75: 2224-2230. 2020.

13. Zhu A, Salminen LE, Thompson PM and Jahanshad N: The UK Biobank Data Parser: A tool with built in and customizable filters for brain studies. Organization for Human Brain Mapping Rome, Italy, June 9-13, 2019. 
14. Cangiano B, Fatti LM, Danesi L, Gazzano G, Croci M, Vitale G, Gilardini L, Bonadonna S, Chiodini I, Caparello CF, et al: Mortality in an Italian nursing home during COVID-19 pandemic: Correlation with gender, age, ADL, vitamin D supplementation, and limitations of the diagnostic tests. Aging (Albany NY) 12: 24522-24534, 2020.

15. Tan CW, Ho LP, Kalimuddin S, Cherng BP, Teh YE, Thien SY, Wong HM, Tern PJ, Chandran M, Chay JW, et al: Cohort study to evaluate the effect of vitamin $\mathrm{D}$, magnesium, and vitamin $\mathrm{B} 12$ in combination on progression to severe outcomes in older patients with coronavirus (COVID-19). Nutrition 79-80: 111017, 2020.

16. Yuan S, Wang R, Chan JF, Zhang AJ, Cheng T, Chik KK, Ye ZW, Wang S, Lee AC, Jin L, et al: Metallodrug ranitidine bismuth citrate suppresses SARS-CoV-2 replication and relieves virus-associated pneumonia in Syrian hamsters. Nat Microbiol 5: 1439-1448, 2020

17. Umhau J: Casting sunlight on an epidemic: Is vitamin $\mathrm{D}$ a critical host factor to prevent COVID-19? J Infect Dis: Mar 25, 2020 (Epub ahead of print).

18. Rubin R: Sorting out whether vitamin D deficiency raises COVID-19 risk. JAMA 325: 329-330, 2021.
19. Murai IH, Fernandes AL, Sales LP, Pinto AJ, Goessler KF Duran CS, Silva CR, Franco AS, Macedo MB, Dalmolin HH, et al: Effect of a single high dose of vitamin D3 on hospital length of stay in patients with moderate to severe COVID-19: A Randomized Clinical Trial. JAMA: Feb 17, 2021. doi: 10.1001/jama.2020.26848.

20. De Flora S, Balansky R and La Maestra S: Rationale for the use of $N$-acetylcysteine in both prevention and adjuvant therapy of COVID-19. FASEB J 34: 13185-13193, 2020.

21. Khanji MY, Aung N, Chahal CA and Petersen SE: COVID-19 and the UK Biobank-Opportunities and challenges for research and collaboration with other large population studies. Front Cardiovasc Med 7: 156, 2020.

This work is licensed under a Creative Commons Attribution-NonCommercial-NoDerivatives 4.0 International (CC BY-NC-ND 4.0) License. 